\title{
S-Modular Games for Distributed Power Allocation in Cognitive Radio Systems
}

\author{
Luca Simone Ronga \\ CNIT, Florence Research Unit \\ Florence, Italy \\ Email: luca.ronga@cnit.it
}

\author{
Enrico Del Re \\ University of Florence and CNIT \\ Florence, Italy \\ Email: enrico.delre@unifi.it
}

\begin{abstract}
Cognitive Radio provides a paradigm to enable an efficient and performing use of spectrum resources. Several challenging issues are still to be solved in order to fully exploit the CR potentials. One of these is the development of efficient distributed power allocation methods to be performed by the independent decisional entities of the network. Game Theory, and in particular S-Modular Games, are attracting since they provide useful tools for the definition of multi objective distributed decisional algorithms in the context of radio communications.
\end{abstract}

\section{INTRODUCTION}

Because of the increasing and continuous demand of services and radio resources, the traditional communication systems which imply an a priori association of the frequency band, the service assigned to it and the used technology, need to become much more flexible, efficient and easy-touse dynamic systems able to cope with the requirements and constraints of the environment and the users. A Cognitive Radio (CR) approach can be considered as a promising and suitable solution to solve this problem. CR systems are characterized by a series of challenging technological problems to be addressed as exposed by Mitola and Haykin in [1], [2]. The search for available resources is not limited to spectrum portions dedicated to unlicensed communications, but is also extended to licensed bands. This paper shows the potential benefits of the adoption of a cognitive radio strategy to the coexistence problem. The developed cognitive radio strategy it has been formulated according the mathematical discipline of Game Theory, with particular reference to SModular Games [3]. The main reason because Game Theory has been considered as resource allocation method is due to its distributed implementation nature. Such attractive feature allows to achieve the flexibility and the efficient adaptation to the operative environment that were previously mentioned. SModular games have been used for power allocation in cellular networks by Altman and Altman in [4]. This paper extends their results in the case a CR dislocation of transmitters and receivers, as well as proposes a new allocation strategy for multi-carrier allocation.

The paper is organized as follows: in Section II the basic theory of S-Modular games is presented. The proposed allocation schemes is reported in Section III, while in Section IV the results from computer simulation are commented. Finally some conclusions are expressed in Section V.

\section{S-Modular GAmes}

Game theory provides a set of powerful tools in order to predict the evolution of dynamical multiple decisional entities operating in the same context. One relevant key classes of games are the supermodular games (S-Games). We focus our attention to a set of $N$ users playing on a strategy space $S$. It means that each element of $S$ represents the possible choice of all players at a given time of the play. Introduced by Topkis in [3], S-Games are normal form games $\Gamma=\left\langle N, S,\left\{f_{i}\right\}\right\rangle$ where $N$ is the set of users, $S$ the strategy space, $f_{i}$ the set of utility functions and $\forall i \in N$ these conditions are satified:

1) the strategy space $S_{i}$ of user $i$ is a complete lattice.

2) $f_{i}$ is supermodular in $s_{i}$.

3) $f_{i}$ presents increasing differences in $x$.

The utility functions $f_{i}$ map the strategy space $S$ on a real value. For convenience the strategy $x \in S$ is decomposed into $\left(x_{i}, x_{-i}\right)$ where $x_{-i}$ indicates all the components of $x$ not belonging to user $i$.

The first condition is easily verified for compact subset of $\Re^{m}$ where $m$ is the strategy space dimension.

Supermodularity of a function $f: X \rightarrow \Re$ states that:

$$
f\left(x^{\prime}\right)+f\left(x^{\prime \prime}\right) \geq f\left(x^{\prime} \vee x^{\prime \prime}\right)+f\left(x^{\prime} \wedge x^{\prime \prime}\right) \quad \forall x^{\prime}, x^{\prime \prime} \in X
$$

Where $a \vee b$ is the join operation defined as the minimum component-wise vector among $a$ and $b$. Similarly $a \wedge b$ is the meet operation defined as the maximum component-wise vector. When $f_{i}$ is twice differentiable, supermodularity is achieved if and only if:

$$
\frac{\partial^{2} f_{i}(x)}{\partial x_{i} \partial x_{j}} \geq 0
$$

for all $x \in S$ and $i \neq j$.

The increasing differences property has been defined in [5]. For a partially ordered set $X \subset \Re^{n}$, we define a relational operator $\geq$ so that we can write $x \geq y$ if $x, y \in X$ and $x_{k} \geq$ $y_{k} \forall k=1, \ldots, n$. A function $f$ has increasing differences in $\left(x_{i}, x_{-i}\right)$ if for all pairs $x_{i}, \bar{x}_{i} \in X_{i}$ such that $x_{i} \geq \bar{x}_{i}$, and all pairs $x_{-i}, \bar{x}_{-i} \in X_{-i}$ such that $x_{-i} \geq \bar{x}_{-i}$, Eq. (3) holds.

$$
f\left(x_{i}, x_{-i}\right)-f\left(\bar{x}_{i}, x_{-i}\right) \geq f\left(x_{i}, \bar{x}_{-i}\right)-f\left(\bar{x}_{i}, \bar{x}_{-i}\right)
$$


The increasing differences property applied to utility functions is relevant to the Cognitive Radio problem. If the strategy space is the allocated power on the radio channels/sub-carriers and the utility function is related to the signal to noise plus interference ratio (SINR) experienced by users, an increased power floor from other users results in a increased power of the desired one.

All the users play with the same strategic rational behavior, following the so-called Best Response (BR) decision rule, defined as for each $i$ :

$$
B R_{i}\left(x_{-i}\right)=\max \left[\arg \max _{x_{i} \in S_{i}} f\left(x_{i}, x_{-i}\right)\right]
$$

The BR decision is a distributed one as it is taken independently from each user. The evaluation of the utility function requires the complete knowledge of the choices operated previously from other users $x_{-i}$. In practical implementations the visibility of others is limited to the effectiveness of the sensing functions available in the Cognitive Radio system as described in [1].

When the cited conditions are met, the S-modular game is proven to have a unique Nash Equilibrium (NE) point and to show a monotone convergence to it when the BR decisions is operated by the users.

A slightly more complicated scenario is found when the strategy set for each user depends from the choices of others, i.e. when $S_{i}=S_{i}\left(x_{-i}\right)$. In this case if the policy set $S_{i}$ is convex and exists a fesible choice $x$ so that $x_{i} \in S_{i}\left(x_{-i}\right)$ and for each user $i, B R_{i}\left(x_{-i}\right)>0$, we obtain the uniqueness of the NE and the convergence to it for the random or roundrobin updating rule.

In the next sections we adapt the above S-modular game to the problem of spectrum coexistence of multiple cognitive radios.

\section{THE PROPOSED ALLOCATION SCHEMES}

The Cognitive Radio context considered in this paper is characterized by $N$ users, namely the players of the game, operating on $M$ radio resources (i.e. different channels or subcarriers of the same multi-carrier channel). Each user is characterized by a transmitter site $T X_{i}$ and a receiver site $R X_{i}$. In the most general context we consider the transmitters and the receivers positions completely independent the ones from the others. The utility function for each user is represented by its instantaneous signal to noise plus interference ratio (SINR), defined as:

$$
\gamma_{i, c}=10 \log _{10}\left(g_{i, i, c} a_{i, c}\right)-10 \log _{10}\left(\sum_{k \neq i} g_{k, i, c} a_{k, c}+\eta_{i}\right)
$$

where $a_{i, c}$ is the power allocated from the transmitter $i$ $\left(T X_{i}\right)$ on the sub-carrier $c, g_{j, i, c}$ is the instantaneous channel response from $T X_{j}$ to $R X_{i}$, while $\eta_{i}$ is the AWGN component at $R X_{i}$. We consider the following utility functions:

$$
u_{i}\left(a_{i}, a_{-i}\right)=\sum_{c=1}^{M}-\left(\gamma_{i, c}\left(a_{i}, a_{-i}\right)-\gamma_{i, c}^{*}\right)^{2}
$$

where $\gamma_{i, c}^{*}$ is the target SINR for user $i$ and sub-carrier $c$. The adopted channel model is composed by a small scale fading and a path-loss component. The path-loss model is the Okomura-Hata model of [6], while the small scale fading is modeled as a Rayleigh process. The utility function represents the (squared) Euclidean distance among the desired SINR vector (in $\mathrm{dB}$ ) and the one obtained after the allocation process. Desired SINR is monotonically related to BER on each particular carrier, so the minimization of the utility results in approaching the desired quality of transmission.

As what concerns the game strategies, we analyzed two possible approaches: the Multiple Carriers Allocation and the Best Carrier Allocation.

\section{A. Multiple Carriers Allocation Strategy}

This decision leads to the selection of the Best Response combination of sub-carriers powers, given the previous allocation of other users. The strategy space is a convex sub-lattice of $\Re^{m}$, i.e. $a_{i} \in S_{i} \in \Re^{m}$ and $0 \leq a_{i, c}<a_{i, M A X}$, for each $c=1, \ldots, M$. A decision is taken by a randomly chosen user at each stage of the game, following the Best Response rule:

$$
B R_{i}\left(a_{-i}\right)=\arg \max _{a_{i} \in S_{i}} u_{i}\left(a_{i}, a_{-i}\right)
$$

\section{B. Best Carrier Allocation Strategy}

With this decision strategy, the selected user first select the best allocation for each possible carrier, then select the carrier where she receives the best response. In other words:

$$
\begin{aligned}
& \forall c \in C_{i} \\
a_{i, c}^{*}= & \arg \max _{a_{i, c} \in S_{i, c}} u_{i}\left(a_{i, c}, a_{i,-c}, a_{-i}\right) \\
B R_{i}^{*}\left(a_{-i}\right)= & \arg \max _{c \in C_{i}} u_{i}\left(a_{i, c}^{*}, a_{i,-c}, a_{-i}\right)
\end{aligned}
$$

where $C_{i}$ is the sub-carriers set, $a_{i, c}$ is the power allocation on carrier $c$ and $a_{i,-c}$ is the power allocation of all the carriers except $c$.

\section{EXPERIMENTAL RESULTS}

To evaluate the performance of the analyzed allocation methods, we focus on the value of the utility function at the end of the game, averaged over the users set. This indicator, recalling the definitions in Eq. (6), represents the mean deviation from the desired SINR: the higher is the value approaching 0 , the better is the allocation obtained by the game.

The operating context is a terrain square area of $1 \mathrm{Km}$ edge. The transmitter positions are chosen randomly in the area, while the receives are placed in a square area of 400 $\mathrm{m}$ around their respective transmitters. An example of the simulated field with 3 users is represented in Fig. 1 where it is also shown in shaded colors the path-loss component of the channel model. The simulations presented here have been conducted with a noise power at the receivers of 0.001 and a $0 \mathrm{~dB}$ power reference for the path-loss component at a distance of 100 meters from the transmitters. 


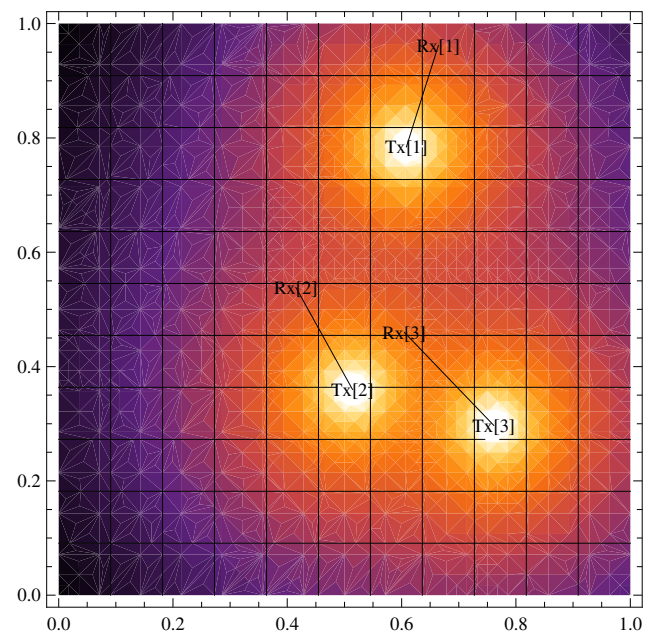

Fig. 1. Instance of a 3 users scenario (shaded colors represent the path loss component of the channel)

\begin{tabular}{|c|c|c||c|}
\hline Users & Carriers & Target SINR & Final Utility \\
\hline 3 & 3 & $5 \mathrm{~dB}$ & $-4.24056 \times 10^{-25}$ \\
\hline 3 & 5 & $5 \mathrm{~dB}$ & $-3.84628 \times 10^{-17}$ \\
\hline 3 & 15 & $5 \mathrm{~dB}$ & -0.00624172 \\
\hline 5 & 3 & $5 \mathrm{~dB}$ & -0.0137993 \\
\hline
\end{tabular}

TABLE I

Results for the Multiple Carrier Allocation Scheme

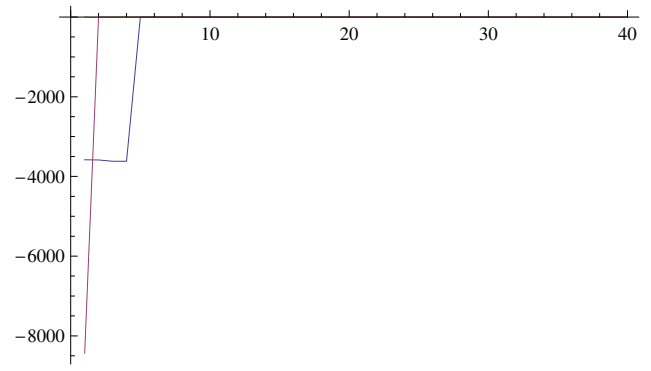

Fig. 2. Convergence of the utility functions for 3-users game with multiple carrier allocation method

Table I shows the final averaged utility functions of the power allocation game for the Multiple Carrier strategy with various configuration of users and carriers.

Table II represents the results for the Best Carrier Allocation Strategy. This method provides similar results with respect to the previous method. Its convergence is assured by S-Modular theory, however in each game iteration a single carrier is allocated, resulting in a slower convergence and a reduced complexity required at the Cognitive Managers.

As expected, the convergence time of the multiple allocation methods is shorter than that of the best carrier selection method. This is shown in the Figs. 2 and 3 where are represented the evolutions of the utility values for a 3 users game (5 sub-carriers, $5 \mathrm{~dB}$ desired SINR) for the first 40 iterations of the game.

As an example, Fig.4 represents a $\Re^{2}$ section of the utility

\begin{tabular}{|c|c|c||c|}
\hline Users & Carriers & Target SINR & Final Utility \\
\hline 3 & 3 & $5 \mathrm{~dB}$ & $-9.15767 \times 10^{-23}$ \\
3 & 5 & $5 \mathrm{~dB}$ & $-4.22214 \times 10^{-18}$ \\
3 & 15 & $5 \mathrm{~dB}$ & -0.631104 \\
\hline 5 & 3 & $5 \mathrm{~dB}$ & $-9.90912 \times 10^{-27}$ \\
10 & 3 & $5 \mathrm{~dB}$ & $-4.7005 \times 10^{-22}$ \\
30 & 3 & $5 \mathrm{~dB}$ & -0.000148154 \\
50 & 3 & $5 \mathrm{~dB}$ & -145.144 \\
\hline 3 & 5 & $10 \mathrm{~dB}$ & -0.02085 \\
3 & 5 & $15 \mathrm{~dB}$ & -1210.41 \\
\hline
\end{tabular}

TABLE II

Results for the Best CARrier Allocation Scheme

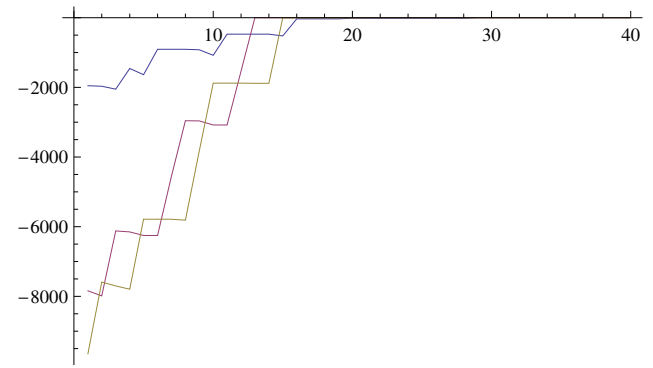

Fig. 3. Convergence of the utility functions for 3-users game with best carrier allocation method

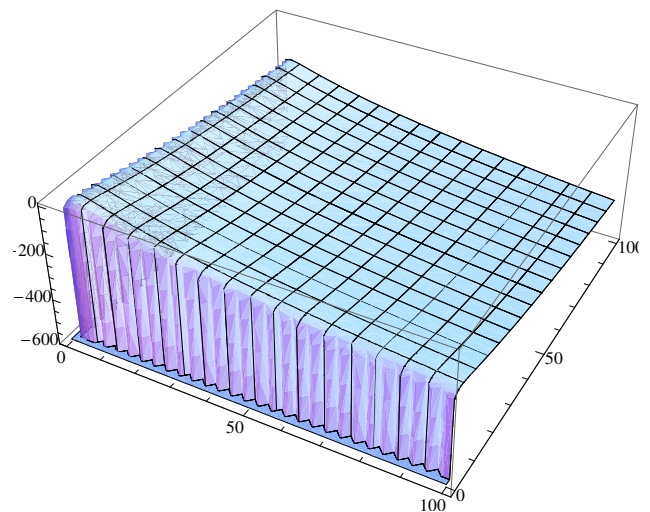

Fig. 4. Utility function section on $\Re^{2}$

function for a specific user of the game with 3 users and 5 resources. In the plot the utility value for the first two carriers at a specific time during the evolution of the game is reported. We can easily recognize the maximum of the utility surface the method will search when the user is allowed to decide. To show the dependence of the allocation from the desired targets, Fig. 5 shows the final averaged value of the utility functions for a 5 users games ( 3 channels, noise variance of $10^{-3}$ ) for a target SINR ranging from 8 to $15 \mathrm{~dB}$. The dashed line refers to the multiple carrier allocation while the continuous line represents the best carrier method. We can note the SINR target regions where the user cannot be satisfied, resulting in a decrease of the utility values. We also note the similar behavior of the two allocation methods. 


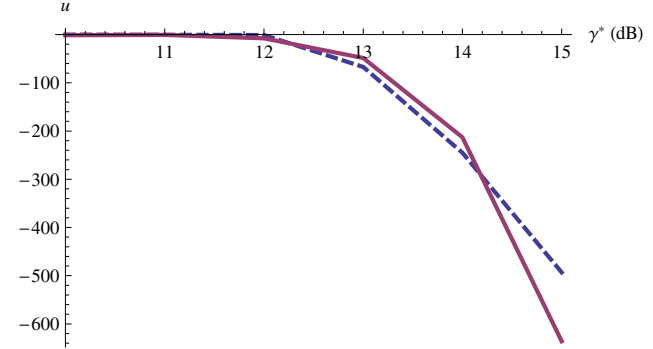

Fig. 5. Averaged utility values vs. SINR for 3 users game (multiple carrier allocation plotted with the dashed line)

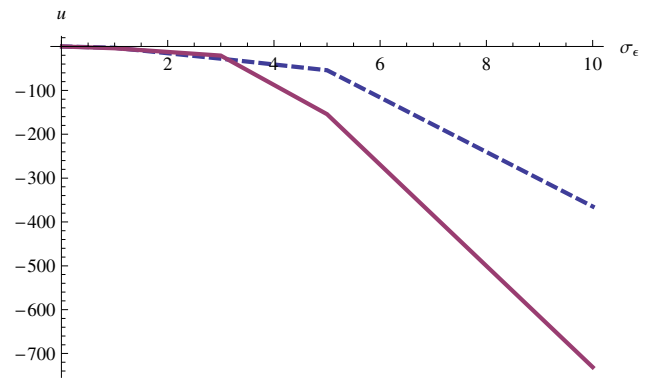

Fig. 6. Effect of power sensing estimate errors on allocation (5 users 3 channels game, $5 \mathrm{~dB}$ of SINR target, multiple carrier allocation plotted with the dashed line)

\section{A. Imperfect Sensing}

In the case of imperfect sensing of the radio environment, the action taken by players lead to a sub-optimal outcome of the game. To model an impaired sensing of the radio context, at each game run, the players can access a "noisy" version of the previous allocation state, given by:

$$
10 \log _{10}(\tilde{a})=10 \log _{10}(a)+\epsilon
$$

where $\epsilon \in \Re^{|S|}$ is the power error vector (in $\mathrm{dB}$ ) whose elements are Gaussian i.i.d. The $|\cdot|$ express the dimension of a space. The Fig. 6 shows the impact of sensing errors on the game evolution. The abscissa represents the standard deviation of the log-power estimate error, while the ordinate is the loss of the averaged true utility function.

\section{B. Complexity and Implementation Considerations}

The proposed scheme is part of the class of distributed resource managers and implies a complete knowledge of the previous allocation step (also derived from sensing). The two presented approaches differ substantially from the complexity point of view. The Multiple Carrier Allocation strategy involves a $M$-dimensional maximization process to be performed by each user at each game step (recall $M$ is the number of carriers). The Best Carrier Allocation is obtained through $M$ one-dimensional maximizations followed by a sorting operation. The drawback of the latter approach is a slower convergence time, as exposed previously in the paper. The complete (though not accurate) knowledge of global allocation status can be obtained through a distributed sensing function. When the deployment area of the CR terminals is large, a local information is sufficient to each terminal so a local un-coordinated sensing can be adopted. On the contrary, in dense distributions of users, a coordinated broadcasted sensing is required.

\section{CONCLUDING REMARKS}

S-Modular Games have applied successfully to the power allocation problem when distributed methods are required. In the CR context, the potentials of this game theory tools are more relevant than in other scenarios due to the desired independence of cognitive transmitters. S-Modular games are proven to be stable, always converging to NE and completely distributed. The proposed methods provide good allocations even in the case of multi-dimensional resource space. Further investigations will analyze the impact of partial information from the radio environment and of computational constraints on the cognitive radio managers.

\section{ACKNOWLEDGMENT}

This work has been supported by Italian Research Program (PRIN 2007) Satellite-Assisted LocalIzation and Communication system for Emergency services (SALICE).

\section{REFERENCES}

[1] S. Haykin, D. J. Thomson, and J. H. Reed, "Spectrum sensing for cognitive radio," Proceedings of the IEEE, vol. 97, no. 5, pp. 849-877, May 2009.

[2] S. Haykin, "Cognitive radio: brain-empowered wireless communications," Selected Areas in Communications, IEEE Journal on, vol. 23, no. 2, pp. 201-220, Feb. 2005.

[3] D. M. Topkis, "Equilibrium points in nonzero-sum $n$-person submodular games," SIAM J. Control Optim., vol. 17, pp. 773-778, 1979.

[4] E. Altman and Z. Altman, "S-modular games and power control in wireless networks," Automatic Control, IEEE Transactions on, vol. 48, no. 5, pp. 839-842, May 2003.

[5] D. Fudenberg and J. Tirole, Game Theory. Cambridge, MA: MIT Press, 1991.

[6] "Urban transmission loss models for mobile radio in the 900 and $1800 \mathrm{mhz}$ bands," European Cooperation in the Field of Scientific and Technical Research EURO-COST 231, The Hague, Tech. Rep. Revision 2, September 1991. 